\title{
Understanding hopping transport and thermoelectric properties of conducting polymers
}

Siarhei Ihnatsenka, Xavier Crispin and Igor Zozoulenko

\author{
Linköping University Post Print
}

Tweet

N.B.: When citing this work, cite the original article.

Original Publication:

Siarhei Ihnatsenka, Xavier Crispin and Igor Zozoulenko, Understanding hopping transport and thermoelectric properties of conducting polymers, 2015, Physical Review B. Condensed Matter and Materials Physics, (92), 3, 035201.

http://dx.doi.org/10.1103/PhysRevB.92.035201

Copyright: American Physical Society http://www.aps.org/

Postprint available at: Linköping University Electronic Press

http://urn.kb.se/resolve?urn=urn:nbn:se:liu:diva-120272 


\title{
Understanding hopping transport and thermoelectric properties of conducting polymers
}

\author{
S. Ihnatsenka, ${ }^{*}$ X. Crispin,$^{\dagger}$ and I. V. Zozoulenko ${ }^{\ddagger}$ \\ Laboratory of Organic Electronics, ITN, Linköping University, SE-60174 Norrköping, Sweden \\ (Received 26 February 2015; revised manuscript received 19 May 2015; published 6 July 2015)
}

\begin{abstract}
We calculate the conductivity $\sigma$ and the Seebeck coefficient $S$ for the phonon-assisted hopping transport in conducting polymers poly(3,4-ethylenedioxythiophene) or PEDOT, experimentally studied by Bubnova et al. [J. Am. Chem. Soc. 134, 16456 (2012)]. We use the Monte Carlo technique as well as the semianalytical approach based on the transport energy concept. We demonstrate that both approaches show a good qualitative agreement for the concentration dependence of $\sigma$ and $S$. At the same time, we find that the semianalytical approach is not in a position to describe the temperature dependence of the conductivity. We find that both Gaussian and exponential density of states (DOS) reproduce rather well the experimental data for the concentration dependence of $\sigma$ and $S$ giving similar fitting parameters of the theory. The obtained parameters correspond to a hopping model of localized quasiparticles extending over 2-3 monomer units with typical jumps over a distance of 3-4 units. The energetic disorder (broadening of the DOS) is estimated to be $0.1 \mathrm{eV}$. Using the Monte Carlo calculation we reproduce the activation behavior of the conductivity with the calculated activation energy close to the experimentally observed one. We find that for a low carrier concentration a number of free carriers contributing to the transport deviates strongly from the measured oxidation level. Possible reasons for this behavior are discussed. We also study the effect of the dimensionality on the charge transport by calculating the Seebeck coefficient and the conductivity for the cases of three-, two-, and one-dimensional motion.
\end{abstract}

DOI: 10.1103/PhysRevB.92.035201

PACS number(s): 72.80.Le, 72.20.Ee, 72.20.Pa

\section{INTRODUCTION}

During recent years the development of technologies using waste heat to produce electricity, such as thermoelectric generators, has been receiving increasing attention [1]. The figure of merit, $Z T=\frac{\sigma S^{2} T}{\kappa}$, describes the efficiency of the thermoelectric power generation; here $\sigma$ is the electrical conductivity, $S$ is the Seebeck coefficient, $\kappa$ is the thermal conductivity, and $T$ is the temperature. The conducting organic polymers have recently emerged as promising materials for thermoelectric applications [1-7]. These materials are cheap, of high natural abundance, and environmentally friendly. A record high figureof-merit $Z T$ value for organic material at room temperature [4-6] has prompted further interest to explore this class of materials for thermoelectric applications aiming at achieving the same $Z T$ value as for the best inorganic materials.

Among all conducting polymers, poly(3,4ethylenedioxythiophene) (PEDOT) has become the material of choice for many applications including thermoelectric ones [8]. This is because PEDOT has a low thermal conductivity, is stable under ambient conditions, is easily processed, has a high electrical conductivity, and can even exhibit a metallic behavior at room temperature $[9,10]$. Despite a massive experimental attention to the electric and thermoelectric transport in PEDOT thin films, many fundamental aspects of charge mobility in this and related materials still remain poorly understood and the interpretation of many experiments remains controversial. It is generally accepted that charge carriers in PEDOT are positively charged spin-carrying polarons and spinless bipolarons resulting from interaction of excess charges with a local

\footnotetext{
*siarhei.ihnatsenka@liu.se

†xavier.crispin@liu.se

†igor.zozoulenko@liu.se
}

distortion of a PEDOT polymer backbone [4,11,12]. It was also shown that a morphology of PEDOT films strongly affects the character of charge dynamics, even though the relative importance and respective role of various structural components (conjugated backbone, counterions, chain breaks, defects, etc.) in determining the carrier mobility remains to be clarified [4,13-19]. Pristine polymeric films show the increase of the conductivity as a function of temperature following the activated [2,3] or stretched exponential dependence $[15,16,19]$. This behavior is attributed to the phonon-assisted hopping transport. Treatment of the pristine PEDOT by solvents or polar compounds leads to the impressive increase of conductivity by several orders of magnitude [4,13-19]. This is also accompanied by the change in the character of the temperature dependence of the conductivity exhibiting a decrease of $\sigma$ as $T$ increases, indicating the insulator-to-metal transition with the bandlike character of charge transport. Note that different studies report different temperature dependence of the high conducting states with microscopic interpretation still being under current debate [15-19].

An efficient control and optimization of the thermoelectric properties of conducting polymers can be achieved using an electrochemical transistor $[3,4,20]$. An active part of this device consists of a conducting polymer channel separated from a gate by an electrolyte with mobile anions and cations. Without an applied gate voltage the channel is in its pristine highly conducting state and the source-drain current $I_{S D}$ is high. An application of the positive gate voltage forces cations from the electrolyte to penetrate the polymer channel. As a result, the channel is gradually reduced (i.e., the concentration of polarons/bipolarons in polymer chains is decreased) resulting in a decrease of $I_{S D}$. The distinct feature of the electrochemical transistor as compared to a conventional organic field-effect transistor [21] is that in the former the electronic transport occurs within the bulk whereas in the latter 
it takes place along an interface. Thus, the electrochemical transistor allows us to probe the bulk thermoelectric properties of an electrochemically active material.

In the present work we focus on the theoretical analysis of the thermoelectric properties of the PEDOT in the electrochemical transistor experimentally studied recently by Bubnova et al. [3]. The activated character of the temperature dependence of the conductivity in the device at hand suggests a thermally assisted hopping. In order to calculate the conductivity and the Seebeck coefficient we perform extensive Monte Carlo calculations complemented by a semianalytical treatment employing a concept of the transport energy [22-24].

The Monte Carlo technique is widely used for calculations of the conductivity in systems with the hopping transport. Following pioneering works of Bässler [25] on simulation of the electronic conductivity in amorphous organic films, a number of studies have utilized the Monte Carlo technique for the calculations of electronic transport in a wide range of polymeric devices including solar cells, light-emitting diodes, and field-effect transistors [26-28]. Note that calculations reported in the above studies correspond to diluted systems out of the thermal equilibrium when the Fermi energy does not enter the theory. On the contrary, thermoelectric properties of conducting polymers are interesting at high oxidation levels (up to $40 \%$ ) for which the system is in the thermal equilibrium and the Fermi level is well defined. In the present work we also utilize the Monte Carlo technique in order to calculate the conductivity and the Seebeck coefficient. It should be mentioned that a standard expression for the Seebeck coefficient widely used in the literature [29,30] is obtained within the relaxation time approximation in the Boltzmann approach, which is appropriate for the band transport. In the present paper we derive an expression for the Seebeck coefficient valid in a general case regardless of the particular mechanism of transport.

It is rather difficult to perform a systematic analysis and fitting of experimental data using the the Monte Carlo method as it requires extensive computational resources. In contrast, such analysis can be easily done using the semianalytical approaches based on the transport energy concept [22-24]. In this paper we compare these two methods and demonstrate that they show a good qualitative and, for some values of parameters, even quantitative agreement. Having established this, we use the semianalytical approach to fit the experimental data of Ref. [3] to extract parameters of the system. This allows us to analyze a shape of the density of states (DOS), as well as to provide a microscopic interpretation of the hopping mechanism identifying an extend of the polaron/bipolaron quasiparticles and their average hopping distance in the structure under consideration. Finally, we investigate how the dimensionality of the motion affects the observed properties of the system and report the results of the Monte Carlo calculations for the Seebeck coefficient and conductivity for the cases of two-dimensional (2D) and one-dimensional (1D) transport.

\section{MODEL}

In order to model the electrical conductivity and the Seebeck coefficient of the system at hand we adopt a standard model of the phonon-assisted hopping widely used for the description of the charge transport in conducting polymers [22-27]. We assume that charge carriers jump between the localized sites of a lattice; at this point we specify neither the precise microscopic structure of the lattice sites nor the nature of the charge carriers. The results of our calculation and the comparison to the experimental data will shed light on these important issues and therefore we postpone a related discussion to Sec. III. The phonon-assisted transition probability between two sites $i$ and $j$ with the energy difference $W=E_{j}-E_{i}$ separated by a distance $R$ is given by the Miller-Abrahams formula [31-33],

$$
v_{i j}=\left\{\begin{array}{ll}
v_{0} \exp \left[-\frac{2 R}{\alpha}-\frac{W}{k T}\right], & W>0 \\
v_{0} \exp \left[-\frac{2 R}{\alpha}\right], & W \leqslant 0
\end{array},\right.
$$

where the localization length $\alpha$ describes an extend of the wave function of a localized state, $k$ is the Boltzmann constant and $v_{0}$ is the intrinsic attempt-to-jump rate, which depends on the strength of the electron-phonon coupling and the phonon density of states.

We assume that charge carriers are in equilibrium and described by the Fermi-Dirac distribution function $f_{F D}(E)$ with the Fermi energy $E_{F}$. Taking into account the occupation probability of the initial state, and nonoccupational probability of the final state, the average transition rate from site $i$ to site $j$ reads $[31,32]$

$$
\Gamma_{i j}=v_{i j} f_{F D}\left(E_{i}\right)\left[1-f_{F D}\left(E_{j}\right)\right] .
$$

Throughout this work we shall neglect an electron-electron interaction, except to allow no more than one electron to occupy a single site.

In our calculations of the conductivity and the Seebeck coefficient we utilize two commonly used shapes of the DOS in conducting polymers, the Gaussian [22-26],

$$
g(E)=\frac{N_{0}}{\sqrt{2 \pi} \sigma_{\mathrm{DOS}}} \exp \left(-\frac{E^{2}}{2 \sigma_{\mathrm{DOS}}^{2}}\right),
$$

and the exponential ones [34],

$$
g(E)=\frac{N_{0}}{2 \sigma_{\text {DOS }}} \exp \left(-\frac{|E|}{\sigma_{\text {DOS }}}\right),
$$

where $N_{0}$ is the concentration of sites, and $\sigma_{\text {DOS }}$ sets a scale for the energetic disorder (the broadening of the DOS). Note that it is still debated in the literature which of these DOS better reproduces the experimental results [24].

We calculate the conductivity $\sigma$ and the Seebeck coefficient $S$ using the Monte Carlo technique and the semi-analytical approach utilizing the so-called transport energy concept. (A detailed description of these approaches is given in Appendixes B and C.) The Seebeck coefficient is given by the expression (see Appendix A),

$$
S(T)=\frac{E_{F}-E_{\text {trans }}}{|e| T},
$$

where the transport energy is defined as the averaged energy weighted by the conductivity distribution

$$
E_{\mathrm{trans}}=\frac{\int E \sigma(E, T)\left(-\frac{\partial f_{F D}}{\partial E}\right) d E}{\sigma(T)},
$$


and the total conductivity at a given temperature being $\sigma(T)=$ $\int d E \sigma(T, E)\left(-\frac{\partial f_{F D}}{\partial E}\right)$.

Note that using the Sommerfeld expansion (see, e.g., Ref. [29]), the Seebeck coefficient, Eqs. (5), (6), can be rewritten in an alternative form

$$
S=-\left.\frac{\pi^{2} k_{B}^{2} T}{3|e|} \frac{\partial}{\partial E} \ln [\sigma(T, E)]\right|_{E=E_{F}} .
$$

This expression (sometimes called the Mott formula) is valid in the limit $k_{B} T \ll E-E_{F}$, where $E$ is the energy of states involved in the conductivity. It follows from the Mott formula that the energy dependence of the Seebeck coefficient is primarily determined by the logarithmic derivative of the DOS at the Fermi energy. Indeed, using the Einstein relation [35,36],

$$
\sigma(T, E)=e^{2} g(E) D(E),
$$

and substituting it into Eq. (7), we obtain,

$$
S \propto \frac{d(\ln g)}{d E}+g \frac{d(\ln D)}{d n} .
$$

Because the diffusion coefficient $D(E)$ is practically independent of the electron density (or only weakly dependent on it), $\frac{d D}{d n} \approx 0$, the Seebeck coefficient is expected to vanish when the DOS has a maximum.

\section{RESULTS AND DISCUSSION}

\section{A. Comparison of the Monte Carlo and the semianalytical approach based on the transport energy concept}

The kinetic Monte Carlo technique provides a direct modeling of the hopping transport in organic semiconductors and therefore it gives the most accurate description of the electronic conductivity. Its disadvantage is that it demands extensive computational resources, which makes it difficult to use this technique to analyze and fit experimental data. In contrast, such analysis can be easily done using the semianalytical approach based on the transport energy concept [22,23] (see Appendix B for its brief description). This approach, however, utilizes various approximations concerning an averaging of the escape rates, hopping distances, etc. To be able to rely on this semianalytical approach for the analysis of the experimental data, in this section we present a comparison of the conductivity and the Seebeck coefficient based on the Monte Carlo (MC) and the semianalytical (sa) methods.

We performed extensive calculations for various values of parameters of the system at hand and we found that the larger the localization length $\alpha$, the better agreement between the Monte Carlo results and the semianalytical calculations based on the transport energy concept. This is illustrated in Fig. 1, which shows calculations for two representative cases of large and small localization lengths, $\alpha=a$ and $\alpha=0.2 a$. We first note that the Monte Carlo calculations based on the definition [Eq. (5)] and on the approximate Mott formula [Eq. (7)] lead to qualitatively similar behavior of the Seebeck coefficient, see Figs. 1(c), 1(d). In both cases $S^{M C}$ exhibits a monotonic growth with energy and, according to the expectations [see Eq. (9)], it vanishes when the DOS reaches a maximum at $E=0$. However, our calculations show that quantitative difference between these two cases can be significant, and therefore in
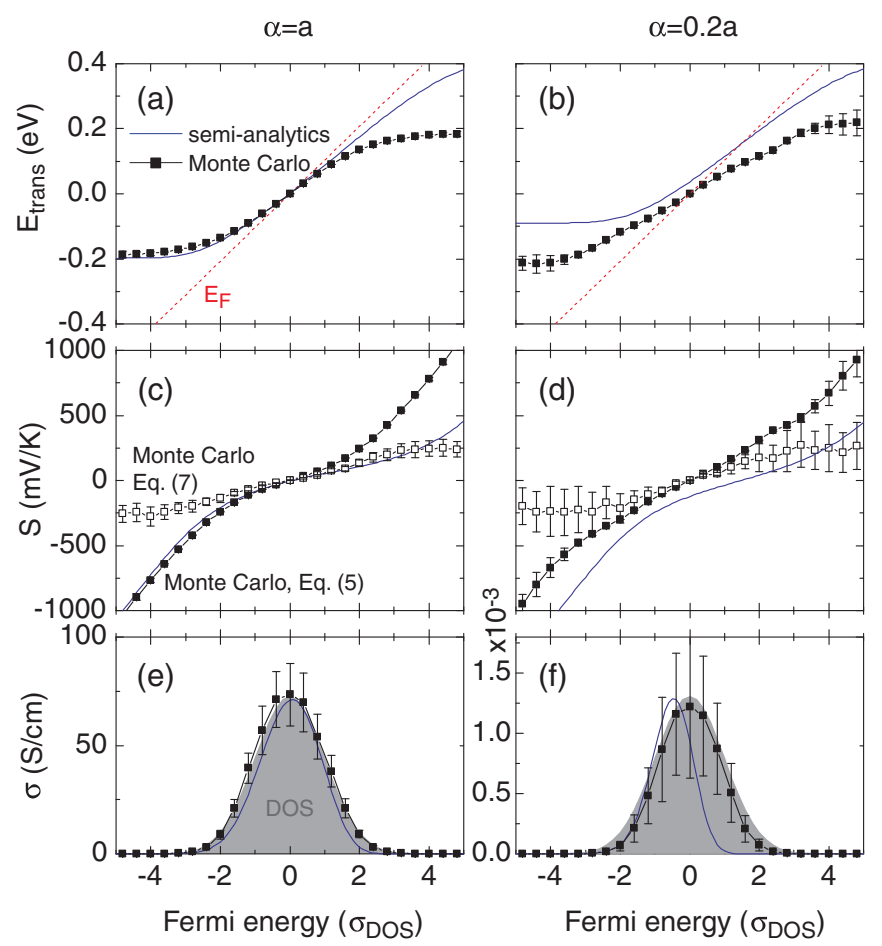

FIG. 1. (Color online) The Monte Carlo and semianalytical calculations of (a) the transport energy $E_{\text {trans }}$, (b) the Seebeck coefficient, (c) the conductivity for different values of the localization length $\alpha=a$ (left), $\alpha=0.2 a$ (right). The DOS is given by the Gaussian, Eq. (3), which is indicated in gray in (e), (f). $\boldsymbol{E}_{\boldsymbol{F}}$ is in units of $\sigma_{\text {DOS }} \cdot \sigma_{\text {DOS }}=4 k T, T=300 \mathrm{~K}$. In semianalytical calculations $v_{0}=$ $10^{12} \mathrm{~s}^{-1}$ was used; for Monte Carlo calculations $v_{0}^{M C}=2.3 \times 10^{13} \mathrm{~s}^{-1}$ (e), $v_{0}^{M C}=4.1 \times 10^{14} \mathrm{~s}^{-1}$ (f). Here and hereafter (unless stated otherwise) the numerical Monte Carlo calculation are performed on the lattice $50 \times 50 \times 50$ with a lattice constant $a=1 \mathrm{~nm}$ and the results are averaged over 16 different samples.

our subsequent discussion we will present results for $S^{M C}$ based on the exact definition, Eq. (5).

Let us now compare $S^{s a}$ and $S^{M C}$. It is seen from Figs. 1(c), 1(d) that they exhibit a qualitative agreement for all values of the parameter $\alpha$. For large $\alpha$ (left panel), $S^{s a}$ and $S^{M C}$ show not only qualitative, but even a relatively good quantitative agreement in the energy interval $E<0$ (corresponding to the relative carrier concentration $\left.n / N_{0} \leqslant 0.5\right)$. For higher energies (and thus for the higher concentrations) the difference between $S^{s a}$ and $S^{M C}$ increases. (Note, however, that such high concentrations are never achieved in experiments.) As the parameter $\alpha$ decreases, the functional dependencies $S^{s a}$ and $S^{M C}$ remain very close to each other, but $S^{s a}$ gets shifted with respect to $S^{M C}$ [Fig. 1(d)].

The difference between $S^{s a}$ and $S^{M C}$ can be traced back to the difference in the corresponding transport energies, $E_{\text {trans }}^{s a}$ and $E_{\text {trans }}^{M C}$, Figs. 1(a), 1(b). For $\alpha=a$ they are close to each other in the energy interval $E<0$. For smaller $\alpha$ the agreement between the transport energies worsens. Note that at low energies the $E_{\text {trans }}$ is practically independent of the electron energy and is situated close to the maximum of the DOS [i.e., close to $E=0$ in Fig. 1(a)] [24]. When the energy is increased such that the position of $E_{F}$ approaches the DOS maximum, 
the transport energy $E_{\text {trans }}$ becomes energy dependent and starts following the position of $E_{F}$. This behavior can be easily understood from the fact that the transport energy plays the role similar to that of the mobility edge [24]. The hopping downward in energy takes place mostly from the states lying above $E_{\text {trans }}$, whereas the hopping upward in energy occurs mostly from the states situated below $E_{\text {trans }}$. As a result, as soon as $E_{F}$ is moved upward and approaches $E_{\text {trans }}$ from below, the latter shifts upward accordingly because otherwise all states below $E_{\text {trans }}$ would be filled and thus unavailable for energy jumps downwards. For higher energies, $E>0$, the difference between $E_{\text {trans }}^{s a}$ and $E_{\text {trans }}^{M C}$ increases, which translates into the corresponding difference between $S^{s a}$ and $S^{M C}$.

Figures 1(e), 1(f) show a comparison of the Monte Carlo and the semianalytical conductivity $\sigma(E)$ as a function of the Fermi energy for the case of the Gaussian DOS, Eq. (3). For the large localization length $(\alpha=a), \sigma^{s c}$ and $\sigma^{M C}$ show not only qualitative but rather a good quantitative agreement. As expected from the Einstein relation, Eq. (8), the conductivity closely follows the DOS. For low localization lengths the agreement between $\sigma^{s c}$ and $\sigma^{M C}$ worsens, see Fig. 1(f). While for energies $E \lesssim 0, \sigma^{s c}$ and $\sigma^{M C}$ remain relatively close to each other, the deviation between them (as well as a deviation between the shapes of $\sigma^{s c}$ and DOS) increases with increase of $E$.

It is important to stress that the semianalytical expression for the conductivity includes a fitting parameter $\eta$ [see Eq. (B4)], which is arbitrarily chosen to adjust the magnitude of the $\sigma^{s c}$. (Note that alternatively one can set $\eta=1$ and adjust a parameter $v_{0}$.) This means that one can only compare the functional dependencies of the conductivities, adjusting the magnitude of $\sigma^{s c}$ by a proper choice of $\eta$ (or $\nu_{0}$ ). In contrast, the semianalytical expression for the Seebeck coefficient does not include this fitting parameter and thus a comparison of $S^{s a}$ and $S^{M C}$ is performed without adjusting the magnitude of $S^{s a}$.

We carried out a comparison of the Monte Carlo and semianalytical approaches for the conductivity for different temperatures and we found that the fitting parameter $\eta$ is not a constant but is temperature dependent, $\eta=\eta(T)$. Its temperature dependence is not provided by the theory. This means that the semianalytical approach is not in a position to describe the temperature dependence of the conductivity. In particular, we find that for large carrier concentration $\sigma^{s c}$ decreases with increase of the temperature, which is in a stark contrast to the experimental findings as well as to the Monte Carlo calculation. Therefore, in the analysis of the temperature dependence of the conductivity reported by Bubnova et al. [3] we will rely on the Monte Carlo results only. It is noteworthy that Li et al. [37] discussed recently the limitations of the semianalytical approaches based on the transport energy concept.

\section{B. Comparison with experimental data}

In this section we will use the semianalytical approach to fit systematically the experimental data reported by Bubnova et al. [3] and to extract the physical parameters of the system such as an extension of the localized state $\alpha$, and the broadening of the DOS $\sigma_{\text {DOS }}$.

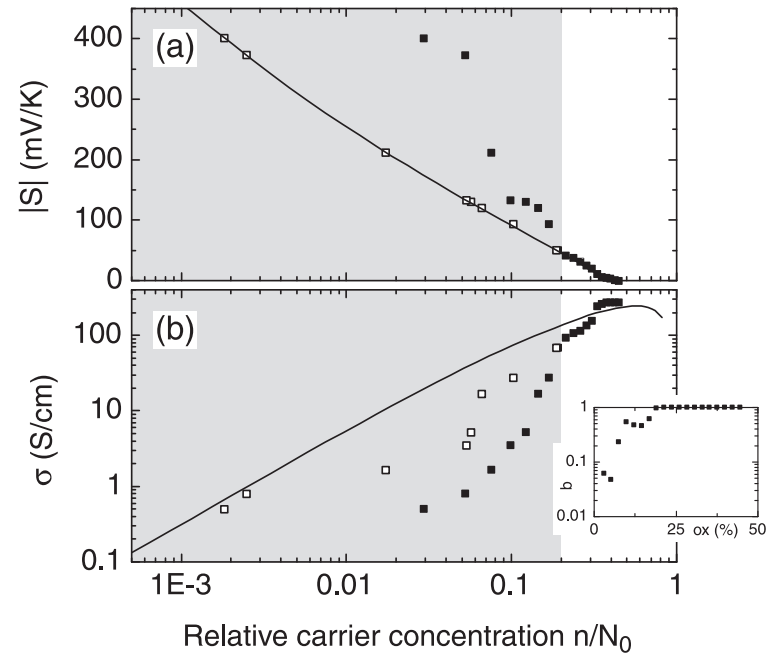

FIG. 2. Comparison of the semianalytical calculations of (a) the Seebeck coefficient and (b) the conductivity with the experimental results of Ref. [3]. Solid lines are semianalytical calculations; filled symbols correspond to the original experimental data as a function of the relative oxidation level $n_{o x}$; open symbols in the grey-shaded region corresponds to experimental data with the rescaled density of free carriers $n=b n_{o x}$ as discussed in the text. The extracted concentration dependence of the parameter $b$ is shown in the inset. $\sigma_{\mathrm{DOS}}=4 k T, T=300 \mathrm{~K}$ (Gaussian DOS is used); $\alpha=1.5 a$, $v_{0}=10^{12} \mathrm{~s}^{-1}$.

Let us first note that the conductivity and the Seebeck coefficient in Ref. [3] are measured as a function of the oxidation level $n_{o x}$. It has been argued by Kim and Pipe [23] that in similar conducting polymers a fraction of free carriers contributing to the transport can be orders of magnitude smaller than the oxidation level if $n_{o x}$ is low. Our results support this conclusion for a PEDOT electrochemical transistor studied in Ref. [3]. Indeed, Fig. 2(a) shows a comparison of the experimental data and the calculated Seebeck coefficient for the Gaussian DOS [Eq. (3)] using parameters $\alpha, \sigma_{\mathrm{DOS}}$, and $v_{0}$ providing the best fit between the theory and experiment in the concentration range $n / N_{0} \gtrsim 0.2$. (A determination of $\alpha, \sigma_{\mathrm{DOS}}$, and $v_{0}$ will be discussed in more detail later in this section.) While for a high relative concentration $n / N_{0} \gtrsim 0.2$ one can achieve a perfect fit between the theory and experiment, no such fit is possible for $n / N_{0} \lesssim 0.2$. Note that we checked that this conclusion holds for other functional dependencies of the DOS, such as an exponential, constant, and power-law DOS. The agreement between the theory and experiment can be restored if we assume that only a fraction of carriers $b=\frac{n}{n_{o x}}$ participates in the transport. Comparing the theoretical curve with the experimentally measured $S$, we extract the density dependence of the parameter $b$ as shown in the inset to Fig. 2(a). The experimental data, rescaled to the effective density $n=b n_{o x}$, is plotted in Fig. 2 using the best fit obtained from the dependence $b=b\left(n_{o x}\right)$.

The difference between the number of free carriers and the oxidation level was attributed by Kim and Pipe [23] to the fact that not all ionized dopants contribute mobile carriers. While this might be true for the material systems discussed in their work, this explanation can hardly be applied 


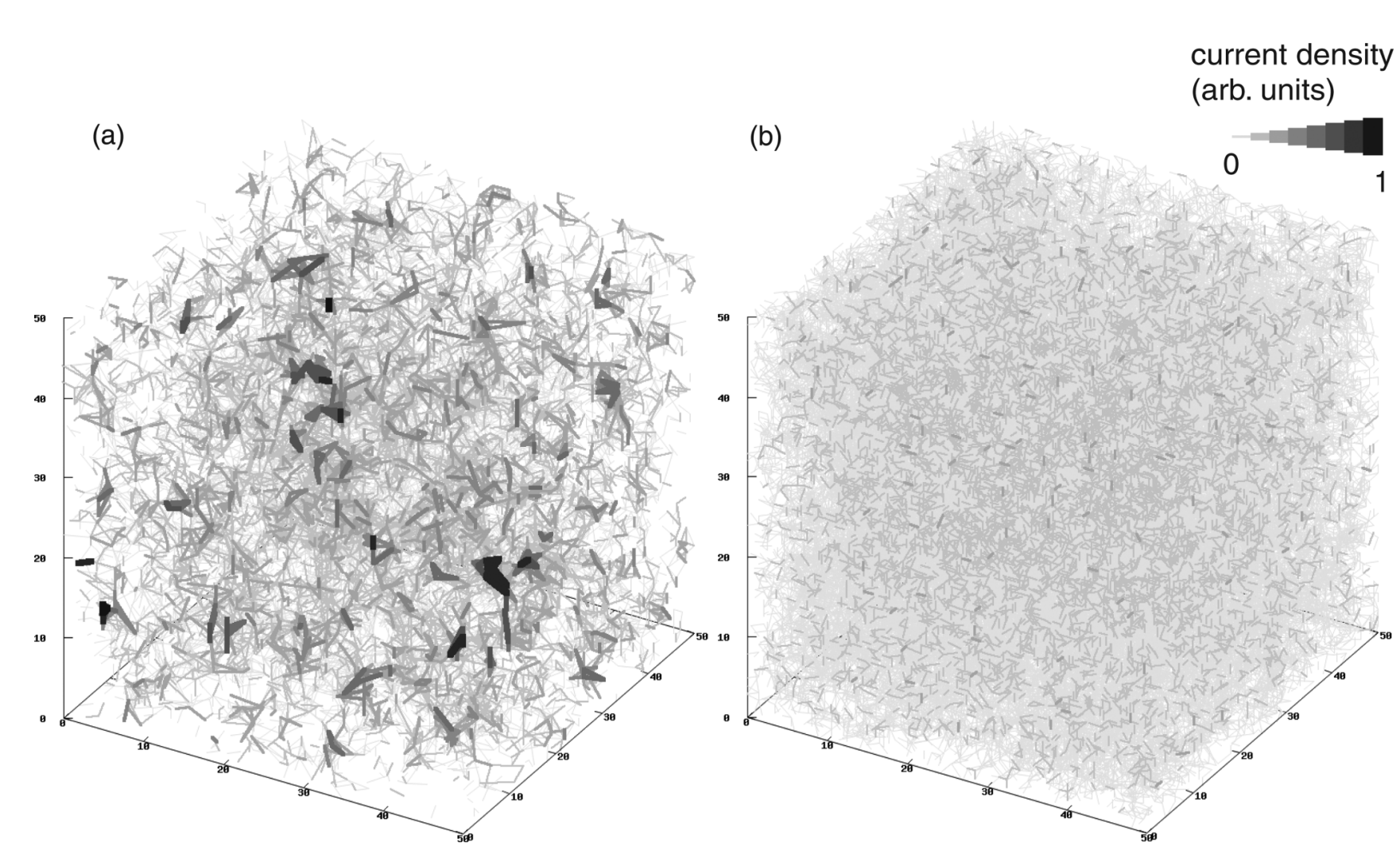

FIG. 3. Visualization of transport paths in $50 \times 50 \times 50$ lattice calculated by the Monte Carlo technique for small and large carrier concentrations, (a) $n / N_{0}=10^{-7}$, (b) $n / N_{0}=0.35$ Temperature $T=300 \mathrm{~K}$; Gaussian DOS with $\alpha=a ; \sigma_{\text {Dos }}=4 k T, T=300$. The magnitude of current is coded by both the thickness and filling as indicated in the inset.

to the electrochemical transistor studied in Ref. [3]. Indeed, in the electrochemical transistor the oxidation level is changed (decreased) by the application of the positive gate voltage to the electrolyte covering PEDOT (see Refs. $[3,4,20]$ for the description of the electrochemical transistor operation). A change of the gate voltage $V_{g}$ does not affect the ionization of dopants (deprotonated sulfonyl groups $\mathrm{SO}_{3}^{-}$). Instead, the positively charged ions $\left(\mathrm{Na}^{+}\right)$are forced from the electrolyte into PEDOT thus reducing the concentration of free carriers (polarons and/or bipolarons) there. Because of the capacitative nature of the gate action, the concentration of positive ions is proportional to the gate voltage and, therefore, the concentration of free carriers (i.e., the oxidation level $n_{o x}$ ) is expected to decrease linearly with the increase of $V_{g}$. The dependence $n=b n_{o x}$ [inset to Fig. 2(a)] shows, however, that for low carrier densities a number of free carriers participating in the transport, $n$, deviates strongly from $n_{o x}$. We speculate that this behavior can be related to the morphology of the material system and the percolative character of the hopping transport in the disordered lattice. To illustrate this we visualize in Fig. 3 charge carriers transport paths in $50 \times 50 \times 50$ lattice calculated by the Monte Carlo technique for large and small carrier concentrations. For the case of the large concentration the transport paths essentially form a homogeneous threedimensional network. As the concentration is reduced, the three-dimensional network gradually transforms into quasione-dimensional percolation chains. With the increase of the concentration of positive ions in the polymer one can expect that an increasing fraction of the charge carriers will be blocked by positive ions in finite chain segments.
Also, the system at hand contains a significant number of carriers executing so-called soft jumps where the carriers are trapped for a very long time within clusters of several sites, which are energetically and/or spatially removed from the remaining sites. These carriers would apparently contribute to the overall oxidation level, but they would not contribute to the transport. An additional reason for the difference between $n$ and $n_{o x}$ can be related to the Andersson localization, which can take place for high disorder concentration (i.e., high concentration of $\mathrm{Na}^{+}$ions) and which can therefore lead to blocking of the transport at the low charge density. More studies are needed in order to resolve this question; work is in progress in order to model the effect of the morphology and the disorder by means of the Monte Carlo and ab initio calculations in the presence of disorder. It would be also interesting to see how the density dependence of the mobility is affected by the fact that the number of carriers contributing to the transport deviates from the measured oxidation level. In particular, more accurate measurements of the actual concentration in experimental samples would help to clarify this issue.

Let us now discuss a determination of the parameters of the theory $\alpha, \sigma_{\mathrm{DOS}}$, and $v_{0}$ as well as the shape of the DOS from the comparison with the experimental data. Because of the difference between $n$ and $n_{o x}$ discussed above, only the interval of the relative carrier concentration $n / N_{0} \gtrsim 0.2$ is used to fit the experimental data; in the remaining interval $n / N_{0} \lesssim 0.2$ we rescale the electron density for the experimental data as described above. We extract $\alpha$ and $\sigma_{\text {DOs }}$ by fitting the semianalytical results for the Seebeck coefficient $S$, and then determine $v_{0}$ using the semianalytical prediction for the 


\section{Gaussian DOS}

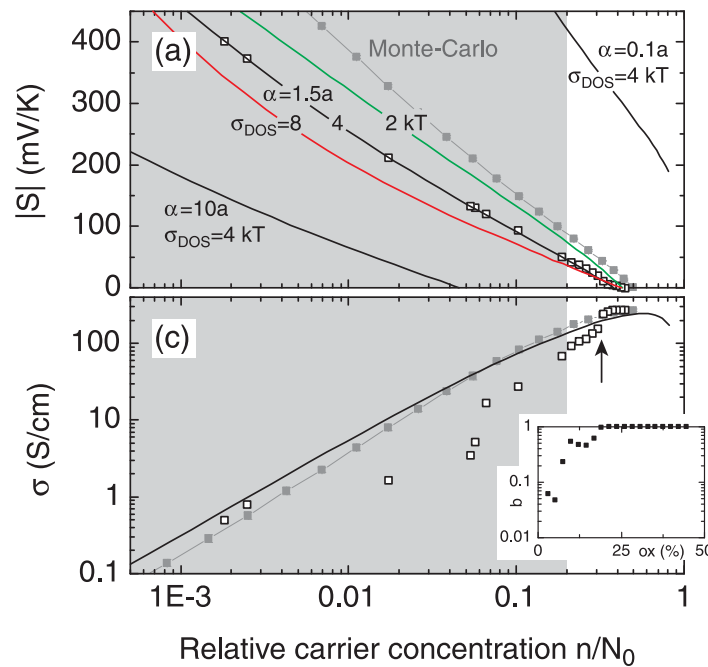

\section{Exponential DOS}
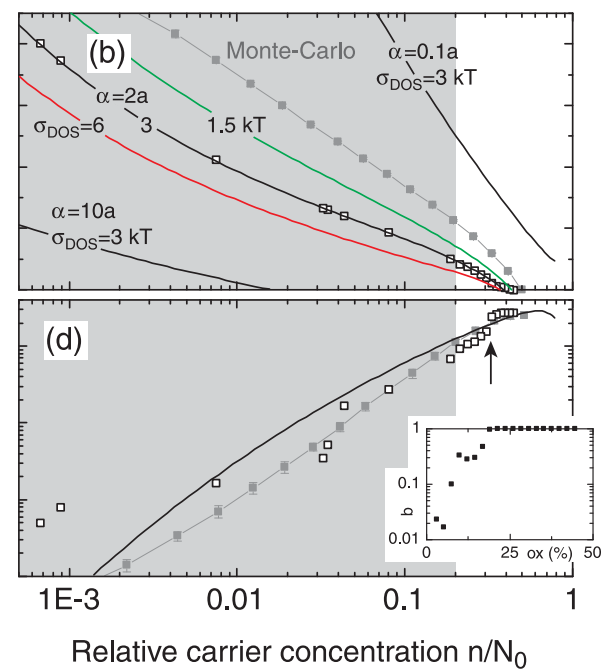

FIG. 4. (Color online) (a)-(b) The Seebeck coefficient $S$ and (c)-(d) the conductivity $\sigma$ as a function of the relative concentration $n / N_{0}$ calculated by the semianalytical model for the Gaussian and exponential DOS. Open squares show experimental data for $S$ and $\sigma$ from Ref. [3]; $v_{0}^{s a}=10^{12} \mathrm{~s}^{-1}$ (a) and $5 \times 10^{11} \mathrm{~s}^{-1}$ (b). The charge carrier density in the experimental data is rescaled as $n=b n_{o x}$; the inset shows the density dependence of the parameter $b$ obtained as outlined in the text (see discussion of Fig. 2). (The gray background corresponds to the density interval for which the experimental data is rescaled). Gray squares and corresponding gray lines show the Monte Carlo calculations with the parameters $\alpha$ and $\sigma_{\mathrm{DOS}}$ corresponding to the best fit for the semianalytical calculations; the Gaussian DOS: $\alpha=1.5 a, \sigma_{\mathrm{DOS}}=4 k T$, $v_{0}^{M C}=1.1 \times 10^{13} \mathrm{~s}^{-1}$; the exponential DOS: $\alpha=2 a, \sigma_{\mathrm{DOS}}=3 \mathrm{kT}, v_{0}^{M C}=3.6 \times 10^{12} \mathrm{~s}^{-1} ; T=300 \mathrm{~K}$. Arrows in (c) and (d) mark concentrations used for the calculation of the temperature dependence of the conductivity shown in Fig. 5.

conductivity $\sigma$. It should be stressed that this fitting provides unambiguous determination of $\alpha$ and $\sigma_{\text {DOS }}$. This is because the variation of $\sigma_{\text {DOS }}$ changes the slope of the Seebeck coefficient, whereas variation of $\alpha$ shifts the curve along the $x$ axis leaving the slope practically unaffected, see Figs. 4(a), 4(c). (Note that $S$ is independent of $v_{0}$ which means that $v_{0}$ can be unambiguously extracted from the conductivity $\sigma$.)

Figures 4(a), 4(b) and 4(c), 4(d) show a comparison of the experimental and theoretical results for the Gaussian and the exponential DOS. Both of them reproduce the experimental data rather well giving similar parameters of the theory, $\alpha=1.5 a, \sigma_{\text {DOS }}=0.1 \mathrm{eV}=4 k T(T=300 \mathrm{~K}), v_{0}=10^{12} \mathrm{~s}^{-1}$ (Gaussian DOS), and $\alpha=2 a, \sigma_{\text {DOS }}=0.075 \mathrm{eV}=3 k T, \nu_{0}=$ $5 \times 10^{11} \mathrm{~s}^{-1}$ (exponential DOS). The values of $\sigma_{\text {DOS }}$ agrees well with the generally assumed disorder strength in organic semiconductors $[25,38]$. The obtained localization length $\alpha$ is an order of magnitude greater than the one typically used in the hopping models for disordered organic materials [26,38]. Large localization lengths obtained from our fitting are in agreement with those reported by Kim and Pipe [23] for pentacene field-effect transistor [39], pentacene films [40], and PEDOT:Tos [4], where they also found $\alpha \approx 1.5-2 a$. In our calculations we used the lattice constant $a=1 \mathrm{~nm}$. Hence, the localization length $\alpha=1.5-2 a$ corresponds to the localized state in the hopping model [Eq. (1)] extended over 2-3 PEDOT monomers (one monomer spans over $\approx 0.8 \mathrm{~nm}$ ). This is similar to a spatial extend of polarons/bipolarons in polymer chains predicted by semiempirical $[41,42]$ and $a b$ initio calculations $[43,44]$. This finding is therefore consistent with a hopping model where the localized states correspond to the polaron/bipolaron quasiparticles extended over several monomer units. It is noteworthy that the obtained pa- rameters of the system correspond to large localization lengths, $\alpha \sim a$, when the agreement between the Monte Carlo and the semianalytical approach is the best (see Sec. III A). The corresponding results of the Monte Carlo simulations with the parameters obtained from the semianalytical fitting are shown for the comparison in Fig. 4.

Note that the experimental results [3] show not only a smooth monotonic decrease (increase) of the Seebeck coefficient (conductivity) as a function of the carrier density, but also some fine structure and bumps in the above dependencies. This fine structure apparently can not be described by a single trial DOS (such as the Gaussian or exponential). We have tried a superposition of several DOS functions and were able to achieve better agreement with the experimental results (not shown here). However, a detailed search for the hopping parameters and the DOS distribution to obtain a quantitative agreement is problematic. In addition, more experimental work is needed to ensure that the observed bumps in the dependencies of $S$ and $\sigma$ are systematic features of PEDOT films. Note that a complicated kinklike DOS dependence was directly measured for the organic tetrathiafulvalene field-effect transistor in Ref. [45]. For conducting polymers OCC-PPV and $\mathrm{P} 3 \mathrm{HT}$ in the regime of the low electron density the shape of the DOS was estimated by Oelerich et al. [46] to be Gaussian. Paulsen and Frisbie [47] have shown that the DOS of the $\mathrm{P} 3 \mathrm{HT}$ in the high-density regime was more complex in shape and can be approximated with no fewer than four Gaussians with numerous heads and shoulders.

Finally, let us discuss the observed temperature dependence of the conductivity. Figure 5 shows a comparison of the Monte Carlo calculations and the experimental results of Bubnova et al. [3]. The experiment shows an activation behavior 

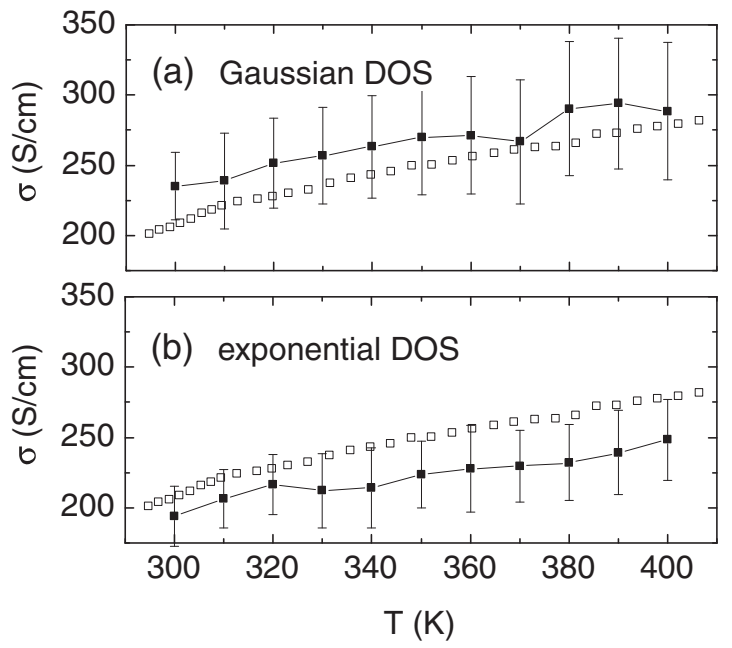

FIG. 5. Monte Carlo calculations of the temperature dependence of the conductivity for the (a) Gaussian and (b) exponential DOS. The experimental data of Ref. [3] is shown by open squares. The parameters $\alpha, \sigma_{\mathrm{DOS}}$, and $v_{0}$ are given in caption to Fig. 4 . The calculations are performed for the relative concentration $\frac{n}{N_{0}} \approx 0.3$ marked by arrows in Fig. 4, which corresponds to the experimental concentration of Ref. [3]. Averaging is done over 96 samples.

$\sigma \propto \exp \left(-E_{a} / k T\right)$ with the activation energy $E_{a}=30.3 \mathrm{meV}$. This behavior is rather well reproduced by the Monte Carlo calculations giving $E_{a}=27.5 .6 \mathrm{meV}$ for the Gaussian DOS and $23 \mathrm{meV}$ for the exponential one.

Note that for lower temperatures the model of hopping transport used in this study represents the basis of the classical variable-range hopping theory developed by Mott and leading to the well-known predictions for the conductivity, $\sigma \propto \exp \left(-\left[T_{0} / T\right]^{1 /(d+1)}\right)$, where $d$ is the dimensionality of the system [31]. At temperatures higher than a certain critical temperature [30] the Mott dependence is replaced by the activation behavior that is reproduced in our study. Shklovskii and Efros [31] have demonstrated that the electron-electron interaction can open the Coulomb gap in the DOS, which leads to the temperature dependence of the conductivity described by $\sigma \propto \exp \left(-\left[T_{C} / T\right]^{1 / 2}\right)$. Note that the ShklovskiEfros dependence can be seen in the conductance at very low temperatures only because the Coulomb gap is smeared by the temperature already at few Kelvin. Recently, a crossover from the Shklovski-Efros dependence to the Mott dependence has been reported for poly(3-hexylthiophene) by Wang et at. [48].

\section{Effect of dimensionality}

We modeled PEDOT structure as a three-dimensional (3D) lattice. It is known that ideal PEDOT crystals form an ordered stacked structure [49,50] with different distances between the planes in different directions. It is also plausible that realistic experimental structures $[3,15,19]$ can be composed of regions with short-range order with dominating transport in two dimensions (within the planes) or in one dimension (along the chains). It is therefore of interest to investigate how the dimensionality of the motion affects the observed properties of the system at hand. In this section we report

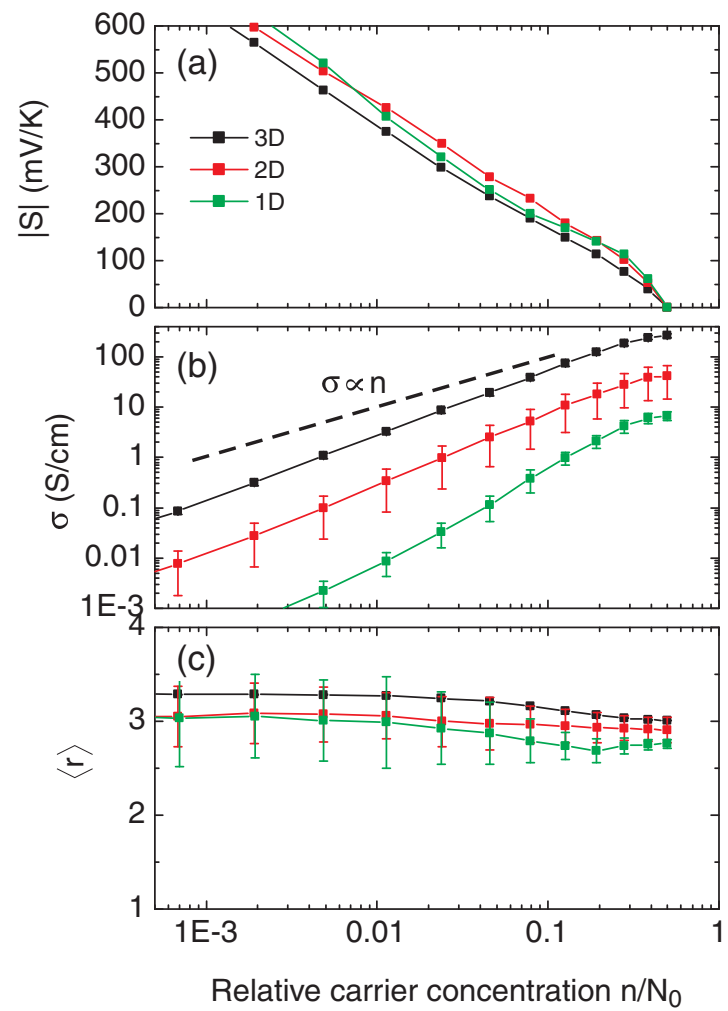

FIG. 6. (Color online) The concentration dependence of (a) the Seebeck coefficient $S$, (b) the conductivity $\sigma$, (c) the average hopping distance $\langle r\rangle$ for the cases of 3D, 2D, and 1D motion calculated using the Monte Carlo technique. Temperature $T=300 \mathrm{~K}$; Gaussian DOS with $\alpha=1.5 a ; \sigma_{\mathrm{DOS}}=4 k T$, and $v_{0}=2.5 \times 10^{12} \mathrm{~s}^{-1} .50 \times 50 \times 50$ lattice was used for $3 \mathrm{D}$ calculations; $50 \times 50$ lattice was used for $2 \mathrm{D}$ calculations; and 50-sites long chain was used for 1D calculations. Dashed line in (b) shows a functional dependence $\sigma \propto n$.

the results of the Monte Carlo calculations for the Seebeck coefficient and conductivity for the cases of two-dimensional (2D) and one-dimensional (1D) motion. The theoretical results allow one to draw a general conclusion on the effect of the reduced dimensionality and might serve as the basis for a further analysis of more complicated morphologies.

The Seebeck coefficient and the conductivity for the cases of 3D, 2D, and 1D motion are shown in Fig. 6 as a function of the carrier concentration. For all cases the conductivity exhibit very similar functional dependencies, close to $\sigma \propto n$ with deviations from this behavior being most pronounced for the 1D case. As expected, for a given concentration the absolute value of the conductivity is largest for the 3D case and decreases as the dimensionality is reduced. The Seebeck coefficients for all dimensionalities show the same functional dependence, and the absolute values of $S$ are rather close for 3D, 2D, and 1D cases. The average hopping distance $\langle r\rangle$ is shown in Fig. 6 . We find that $\langle r\rangle \approx 3 a$ being practically independent on both $n$ and dimensionality. For the system at hand this hopping distance corresponds to jumps over 3-4 PEDOT unit cells.

We mentioned above that in the variable range hopping regime (corresponding to the case of the hopping transport at low temperatures) the temperature dependence of the 
conductivity is different for the cases of $3 \mathrm{D}, 2 \mathrm{D}$, and $1 \mathrm{D}$ transport [31]. This allows one to draw a conclusion about the dimensionality of the system under study from the temperature measurements. Our findings demonstrate that a concentration dependence of the Seebeck coefficient and conductivity can not be used to obtain information about the dimensionality of the transport. On the other hand, the insensitivity of the functional dependence $S=S(n)$ and $\sigma=\sigma(n)$ on the dimensionality justifies the use of 3D lattice for the Monte Carlo calculations.

\section{CONCLUSIONS}

In this work we present a theoretical analysis of the thermoelectric properties of the electrochemical transistor reported by Bubnova et al. [3]. In order to calculate the conductivity and the Seebeck coefficient for the system at hand we adopt a standard model of the phonon-assisted hopping transport and perform extensive Monte Carlo calculations complemented by a semianalytical treatment employing the concept of the transport energy.

Our main findings can be summarized as follows:

(i) We perform the Monte Carlo calculation of the Seebeck coefficient for the hopping transport in a disordered organic material. We find that the Monte Carlo and the semianalytical approaches show a good qualitative agreement for the concentration dependence of the conductivity and the Seebeck coefficient. We find that the above agreement depends primarily on the localization length $\alpha$ in the hopping model: the larger $\alpha$, the better the agreement. In particular, we find that when the localization length is of the order of the lattice constant, the agreement between the Monte Carlo and the semianalytical approaches becomes almost quantitative. At the same time, we find that the semianalytical approach is not in a position to describe the temperature dependence of the conductivity.

(ii) In contrast to the exact Monte Carlo calculations, the semianalytical approach does not demand extensive computational resources. This allows us to use this approach to perform a systematic analysis of the experimental data and extract parameters of the system at hand. Using this approach to extract the experiment data from the concentration dependence of the the Seebeck coefficient and conductivity we find that both Gaussian and exponential DOS reproduce the experimental data rather well giving similar parameters of the theory. In particular, we find that the localization length $\alpha \approx 1.5 \mathrm{~nm}$, disorder strength $\sigma_{\mathrm{DOS}} \approx 0.1 \mathrm{eV}=4 k T$ (at $T=$ $300 \mathrm{~K}$ ) and attempt-to-escape frequency $v_{0} \approx 10^{12} \mathrm{~s}^{-1}$. The average hopping distance obtained from the Monte Carlo calculations is $\langle r\rangle \approx 3 \mathrm{~nm}$. The fitting of the experimental data suggests therefore a hopping model where localized states correspond to the polaron/bipolaron quasiparticles extended over 2-3 PEDOT monomer units with typical jumps over a distance of 3-4 monomer units.

(iii) We find that for a low carrier concentration a number of free carriers contributing to the transport deviates strongly from the measured oxidation level. While this finding is in agreement with the previous result reported for similar conducting polymers, for the electrochemical transistor studied in Ref. [3] we propose here an alternative interpretation. (iv) Using the Monte Carlo calculation we reproduce the activation behavior of the conductivity with the calculated activation energy close to the experimentally observed one.

(v) We study the effect of the dimensionality on charge transport calculating the Seebeck coefficient and the conductivity for the cases of 3D, 2D, and 1D motion. For all cases the conductivity exhibits very similar functional dependence, close to $\sigma \propto n$; a deviation from this behavior is most pronounced for the 1D case. The Seebeck coefficients for all dimensionalities also show the same functional dependence, and the absolute values of $S$ are rather close for 3D, 2D, and $1 \mathrm{D}$ cases.

(vi) The expressions for the Seebeck coefficient $S$ available in the literature $[29,30,32]$ are usually derived within the relaxation time approximation in the assumption of the band transport. In this study we present a general derivation of the Seebeck coefficient without a relation to any particular mechanism of transport.

\section{ACKNOWLEDGMENTS}

We are grateful to M. Kemerink for helpful discussions. The research was supported by the Energimyndigheten, the European Research Council (ERC-starting-grant 307596), and the Knut and Alice Wallenberg Foundation (The Tail of the Sun).

\section{APPENDIX A: DERIVATION OF THE SEEBECK COEFFICIENT $S$}

Expressions for the Seebeck coefficient $S$ available in the literature $[29,30,32]$ are usually derived within the relaxation time approximation in the assumption of the band transport. It is therefore not a priori evident how and whether these expressions can be used for the description of the hopping motion. In this appendix we present a general derivation of the Seebeck coefficient without a relation to any particular mechanism of transport including the hopping one.

We start by expressing the Seebeck coefficient via the electrical current, $J$, and the energy current, $J_{\varepsilon}[29,30,32]$,

$$
S=\frac{J_{\varepsilon}-\mu J}{-|e| T J} .
$$

Express $J$ and $J_{\varepsilon}$ in a standard way as $J \equiv\langle J\rangle=\operatorname{Tr}\{\rho \hat{J}\}$, $J_{\varepsilon} \equiv\left\langle J_{\varepsilon}\right\rangle=\operatorname{Tr}\left\{\rho \hat{J}_{\varepsilon}\right\}$, where $\rho$ is the statistical operator for the Hamiltonian $\hat{H}, \rho=\frac{1}{Z} e^{-\frac{\hat{H}}{k T}}, Z=\sum_{n} e^{-\frac{E_{n}}{k T}},\left(\hat{H}|n\rangle=E_{n}|n\rangle\right)$, and the quantum-mechanical particle and energy currents are $\hat{J}=\hat{J}_{k}=\frac{1}{V} v_{k}, \hat{J}_{\varepsilon}=\varepsilon_{k} \hat{J}_{k}=\frac{1}{V} \varepsilon_{k} v_{k}[29,32]$ ( $V$ is the volume, $v$ is the velocity).

Consider a perturbed system, $\rho=\rho_{0}+\delta \rho$, where $\rho_{0}$ corresponds to the equilibrium situation when no current flows, $\operatorname{Tr}\left\{\rho_{0} \hat{J}\right\}=0$. (Note that $\rho_{0}\left|\psi_{E}\right\rangle=f_{F D}\left|\psi_{E}\right\rangle$, where $f_{F D}$ stands for the Fermi-Dirac distribution $f_{F D}=$ $\left.\frac{1}{1+\exp \left[\left(E-F_{F}\right) / k T\right]}.\right)$ Then $\operatorname{Tr}\{\rho \hat{J}\}=\sum_{k k^{\prime}}\left\langle k|\delta \rho| k^{\prime}\right\rangle\left\langle k^{\prime}\left|\hat{J}_{k}\right| k\right\rangle=$ $\frac{1}{V}|e| T \iint d E d E^{\prime} g(E) g\left(E^{\prime}\right)\left\langle E^{\prime}|\delta \rho| E\right\rangle\left\langle E|v| E^{\prime}\right\rangle$, and Eq. (A1) reads,

$$
S=-\frac{\iint d E d E^{\prime} g(E) g\left(E^{\prime}\right)\left\langle\psi_{E^{\prime}}|\delta \rho| \psi_{E}\right\rangle\left\langle\psi_{E}|(E-\mu) v| \psi_{E^{\prime}}\right\rangle}{|e| T \iint d E d E^{\prime} g(E) g\left(E^{\prime}\right)\left\langle\psi_{E^{\prime}}|\delta \rho| \psi_{E}\right\rangle\left\langle\psi_{E}|v| \psi_{E^{\prime}}\right\rangle},
$$


where $g(E)$ is the density of states (DOS), and $\psi_{E}$ is the eigenfunction of the Hamiltonian $\hat{H}$ at the energy $E$.

Let us now specify the perturbation of the system. Assuming a harmonic variation of the form $\delta \rho(t)=\delta \rho e^{-i \omega t+\alpha t}, \delta \hat{H}(t)=\delta \hat{H} e^{-i \omega t+\alpha t}$ (and letting $\alpha \rightarrow 0$ ) one can show that [32]

$$
\left\langle\psi_{E^{\prime}}|\delta \rho| \psi_{E}\right\rangle=\frac{f_{F D}\left(E^{\prime}\right)-f_{F D}(E)}{E^{\prime}-E-\hbar \omega-i \hbar \alpha}\left\langle\psi_{E^{\prime}}|\delta \hat{H}| \psi_{E}\right\rangle .
$$

The system responses to the change of the electric field $F$, which is, in turn, related to the vector potential $A=A_{0}+\delta A$ as $F=-\frac{\partial}{\partial t}(\delta A)$, such that $\delta \hat{H}=-\frac{|e| F v}{i \omega}$ [32]. Using the representation of the $\delta$ function $\operatorname{Re}\left(\lim _{\alpha \rightarrow 0} \frac{1}{E^{\prime}-E-\hbar \omega-i \hbar \alpha}\right)=$ $i \pi \delta\left(E^{\prime}-E-\hbar \omega\right)$ and considering limit $\omega \rightarrow 0$ when $\frac{f_{F D}(E+\hbar \omega)-f_{F D}(E)}{\hbar \omega}=\frac{\partial f_{F D}}{\partial E}$, we obtain from Eqs. (A2), (A3)

$$
\begin{gathered}
S(T)=-\frac{1}{|e| T} \frac{\int d E\left(E-E_{F}\right) \sigma(T, E)\left(-\frac{\partial f_{F D}}{\partial E}\right)}{\sigma(T)}, \\
\sigma(T)=\int d E \sigma(T, E)\left(-\frac{\partial f_{F D}}{\partial E}\right), \\
\sigma(T, E)=2 e^{2} \pi \hbar V|g(E)|^{2}\left\langle\psi_{E}|v| \psi_{E}\right\rangle^{2},
\end{gathered}
$$

where factor 2 in Eq. (A6) accounts for spin. Note that expressions for $S(T)$ and $\sigma(T)$, Eqs. (A4), (A5), are formally identical to corresponding equations in Ref. [29] (ch. 13) where, however, $\sigma(T, E)$ is expressed via Boltzmann's relaxation time (which is appropriate for the case of band transport). We point out that our expression for $\sigma(T, E)$ [given by Eq. (A6)] is not limited to any particular mechanism of transport.

Let us focus on the expression for the conductivity, Eq. (A6), and rewrite it in alternative forms corresponding to the Kubo-Greenwood formula and the Einstein relation. Substituting Eq. (A6) into Eqs. (A4), (A5) and changing an integration over energy into summation over all available states $k$ we obtain for the nominator and denominator of Eq. (A4) (upper and lower rows in the square brackets correspondingly),

$$
\begin{aligned}
2 e^{2} & \pi \hbar \int d E\left[\begin{array}{c}
\left.\left(E-E_{F}\right)\right] \\
1
\end{array}\right]|g(E)|^{2}\left\langle\psi_{E}|v| \psi_{E}\right\rangle^{2}\left(-\frac{\partial f_{0}}{\partial E}\right) \\
= & \frac{2 e^{2} \pi \hbar}{V} \sum_{k, k^{\prime}} v_{k k^{\prime}} v_{k^{\prime} k}\left[\begin{array}{c}
\left.\left(E_{k}-E_{F}\right)\right] \delta\left(E_{k}-E_{k^{\prime}}\right)\left(-\frac{\partial f_{0}}{\partial E}\right) \\
1
\end{array}\right] \\
= & \frac{2 e^{2} \pi \hbar}{V} \int_{-\infty}^{\infty} d E\left[\begin{array}{c}
\left(E-E_{F}\right) \\
1
\end{array}\right] \sum_{k, k^{\prime}} v_{k k^{\prime}} v_{k^{\prime} k} \\
& \times \delta\left(E-E_{k}\right) \delta\left(E-E_{k^{\prime}}\right)\left(-\frac{\partial f_{0}}{\partial E}\right) \\
= & \frac{2 e^{2} \pi \hbar}{V} \int_{-\infty}^{\infty} d E\left[\begin{array}{c}
\left(E-E_{F}\right) \\
1
\end{array}\right] \operatorname{Tr}[v \delta(E-\hat{H}) v \delta(E-\hat{H})] \\
& \times\left(-\frac{\partial f_{0}}{\partial E}\right),
\end{aligned}
$$

where in Eq. (A9) we utilized the identity $\delta\left(E_{k}-E_{k^{\prime}}\right)=$ $\int_{-\infty}^{\infty} d E \delta\left(E-E_{k}\right) \delta\left(E-E_{k^{\prime}}\right)$, and in Eq. (A10) we used a definition of the trace. It follows from Eq. (A10) that the definition of $\sigma(T, E)$ in Eqs. (A4)-(A6) can be reduced to the standard Kubo-Greenwood formula [32],

$$
\sigma(T, E)=\frac{2 e^{2} \pi \hbar}{V} \operatorname{Tr}[v \delta(E-\hat{H}) v \delta(E-\hat{H})] .
$$

It is worth mentioning that the Kubo-Greenwood formula transforms to a familiar expression for the conductivity corresponding to the Einstein relation $[35,36]$,

$$
\sigma(T, E)=e^{2} g(E) D(E)
$$

where the diffusion coefficient is given by the mean quadratic spreading

$$
D(E)=\lim _{t \rightarrow \infty} \frac{\left\langle(\hat{X}(t)-\hat{X}(0))^{2}\right\rangle}{t},
$$

with $\hat{X}(t)$ being the position operator in the Heisenberg representation.

\section{APPENDIX B: SEEBECK COEFFICIENT IN THE SEMIANALYTICAL APPROACH WITHIN THE TRANSPORT ENERGY CONCEPT}

In this appendix we briefly outline formulas that we use to calculate the Seebeck coefficient within the transport energy concept based on the results of Schmechel et al. [22]; note that a similar approach was also used by Kim and Pipe [23].

Start by introducing the (differential) escape rate distribution $v_{e s c}^{\prime}\left(E_{0}, W\right)$ for an electron on the energy $E_{0}+W$ (where $E_{0}$ is the initial state),

$$
v_{e s c}^{\prime}\left(E_{0}, W\right)=\frac{v_{0}}{k T} \exp \left[-2 \alpha \bar{R}\left(E_{0}+W\right)-\frac{W}{k T}\right],
$$

where $\bar{R}(E)$ is the mean distance that has to be overcome by an electron of energy $E$ by tunneling, $\bar{R}(E)=$ $\left(\frac{4 \pi}{3 B} \int_{-\infty}^{E} g(\varepsilon)\left[1-f_{F D}(\varepsilon)\right] d \varepsilon\right)^{-1 / 3}$. In the last expression $B$ is a parameter that accounts for a percolative character of hopping transport in a disordered system; it was chosen $B=1$ in Ref. [22] and $B=2.7$ in Ref. [23]. Introduce the differential conductivity $\sigma^{\prime}(E)=e n^{\prime}(E) \mu(E)$ and the differential particle density $n^{\prime}(E)=g(E) f_{F D}(E)$ such that

$$
\begin{gathered}
\sigma=\int_{-\infty}^{+\infty} \sigma^{\prime}(E) d E \\
n=\int_{-\infty}^{+\infty} n^{\prime}(E) d E .
\end{gathered}
$$

[As follows from Eq. (A5), the relation between the differential conductivity and the conductivity $\sigma(E, T)$ used in the Monte Carlo calculations [Eqs. (A6), (A8), (A12)] is $\sigma^{\prime}=\sigma(T, E)\left(-\frac{\partial f_{F D}}{\partial E}\right)$.] The mobility $\mu(E)$ is given by the generalized Einstein relation

$$
\mu(E)=\frac{|e|}{k T}\left(1-f_{F D}\right) D(E) \eta,
$$


where $\eta$ is a fitting constant, and the diffusion coefficient is given by

$$
D(E)=\lambda^{2}(E) v_{e s c}(E),
$$

where $\lambda(E)=\bar{R}\left[E_{\text {esc }}(E)\right]$ is the carrier mean jump distance at energy $E, v_{\text {esc }}(E)$ is the total escape rate of an electron from the state with the energy $E$

$$
v_{e s c}(E)=\int_{0}^{+\infty} v_{e s c}^{\prime}(E, \varepsilon) d \varepsilon,
$$

$E_{\text {esc }}(E)$ is the mean energy at which a carrier is released from an initial state at the energy $E$,

$$
E_{e s c}(E)=E+\frac{\int_{0}^{+\infty} \varepsilon v_{e s c}^{\prime}(E, \varepsilon) d \epsilon}{\int_{0}^{+\infty} v_{e s c}^{\prime}(E, \varepsilon) d \epsilon} .
$$

It is noteworthy that the definition of the diffusion coefficient, Eq. (B5), is consistent with the definition based on Eq. (A13) where the square of the mean jump length during time $\tau=v_{\text {esc }}^{-1}$ corresponds to the mean quadratic spreading during time $t$.

Finally, the Seebeck coefficient is given,

$$
S(T)=-\frac{1}{|e| T} \frac{\int d E\left(E-E_{F}\right) \sigma^{\prime}(E)}{\sigma(T)}=\frac{E_{F}-E_{\text {trans }}}{|e| T},
$$

where the transport energy $E_{\text {trans }}$ is the averaged energy weighted by the conductivity distribution,

$$
E_{\text {trans }}=\frac{\int \varepsilon \sigma^{\prime}(\epsilon) d \varepsilon}{\sigma(T)} .
$$

\section{APPENDIX C: KINETIC MONTE-CARLO METHOD}

This section describes the main steps in the simulation of the hopping transport based on the kinetic Monte Carlo method [25,26,51]. The method includes a definition of a lattice, assigning energy levels to every site according to the chosen DOS, and then running the simulation by monitoring a random walk of charge carriers between the sites. In our calculations we do not account for electron-electron interaction except allowing no more than one electron to occupy a single site.

The organic semiconductor is modeled by a 3D $N_{x} \times N_{y} \times$ $N_{z}$ lattice with a lattice constant $a$ where the periodic boundary conditions are chosen in all directions. For a given lattice configuration a random walk of typically 10 charge carriers is simulated. This is known to be a reasonable compromise between computer time and computational statistics [25]. It is worth noting that in our model the Fermi energy is explicitly defined, which means that the charge carrier concentration is given not by the above number of carriers but by the number of sites occupied according to the Fermi-Dirac statistics for a given $E_{F}$. Several lattice configurations (typically 16 ) with different disorder realizations are used to obtain statistically averaged quantities of $\sigma$ and $S$.

In order to generate a current we apply a small potential difference $V$ to the boundaries of the sample in the $x$ direction $\left(|e| V=k_{B} T / 10\right)$. The energy on the site with the coordinate $x_{i}$ transforms into $E_{i} \rightarrow E_{i}-|e| \frac{V}{L_{x}} x_{i}$, where $L_{x}$ is the size of the computational domain in the $x$ direction. Because we assume a local equilibrium, the Fermi energy drops linearly between $E_{F}^{\text {left }}$ and $E_{F}^{\text {right }}=E_{F}^{\text {left }}-|e| \frac{V}{L}$. Apparently, with this transformation, the difference $E_{i}-E_{F}$ remains locally unchanged under an application of the external voltage.
The kinetic Monte Carlo simulation proceeds as follows.

(i) Initialization of site energies $E_{i}$. For every site $i$, a random value of the energy is drawn from a Gaussian or exponential DOS, Eqs. (3), (4). To facilitate generation of the random energies the Metropolis-Hastings algorithm is used.

(ii) Initial placement of charges. $N_{\text {charges }}$ charges whose dynamics will be explicitly traced are randomly placed on the lattice according to the Fermi-Dirac occupation probabilities.

(iii) Choice of hopping events. First, using Eq. (1), we calculate all the hopping rates $v_{i j}$ from the sites $i$ where charges have been placed on the previous step. To prevent hopping into already occupied sites, we set corresponding hopping rates equal to 0 . Because the hopping rate decreases exponentially with the distance, it is possible to introduce a cut-off distance and set $v_{i j}=0$ for the jumps longer than this distance. This reduces substantially the computational time and resources. We have checked that for the parameters and regimes studied in the present work, practically no jumps occur between sites separated by the distance $6 a$ and we thus use this value as the cut-off distance.

We renormalize the hopping rates $\Gamma_{i j}$ introducing corresponding probabilities, $p_{i j}$,

$$
p_{i j}=\frac{\Gamma_{i j}}{\sum_{i^{\prime} j^{\prime}} \Gamma_{i^{\prime} j^{\prime}}} .
$$

In the above sum we include only jumps from the occupied to unoccupied sites, i.e., we set $\Gamma_{i j}=0$ if site $j$ is occupied or site $i$ is empty. Then, for each charge, we randomly choose a hopping event with a probability equal to Eq. (C1) using a following algorithm. First, we enumerate all hopping events introducing for every pair $i j$ an index $k$ (i.e., $i j \rightarrow k$, and $p_{i j}=p_{k}$ ), where $k \in\left\{1, \ldots, k_{\max }\right\}$, with $k_{\max }$ being the total number of all possible hopping events. Then a partial sum $S_{k}$ is defined for every index $k$

$$
S_{k}=\sum_{k^{\prime}=1}^{k} p_{k^{\prime}} .
$$

Apparently, for every $k$ the length of the interval $\left[S_{k-1}, S_{k}\right]$ is equal to the probability $p_{k}$ for the $k$ th jump, and the total length of all intervals is equal to 1 , i.e., $S_{k_{\max }}=1$. Then we draw a random real number $r$ from the interval $[0,1]$ and find the index $k$ such that $S_{k-1} \leqslant r \leqslant S_{k}$, which gives us the hopping event that will occur. Having chosen the hoping event we move a charge between the corresponding sites $i$ and $j$.

(iv) Calculation of the waiting time. After every hopping event, we add to the total simulation time $t$ the waiting time $\tau$ that has passed until the event took place. This time is obtained by drawing a random number from the exponential waiting time distribution $P(\tau)=v_{i} \exp \left[-v_{i} \tau\right]$ with $v_{i}=\sum_{j} v_{i j}$ being the total hopping rate of hopping from site $i$. It is therefore given by

$$
\tau=-\frac{1}{v_{i}} \ln r,
$$

where a random number $r$ is drawn from the interval $[0,1]$.

(v) Calculation of the current density. Every time that a predefined number of jumps has occurred, we calculate the 
current density, $J(t)$, via the following expression

$$
J(t)=\frac{e\left(N^{+}-N^{-}\right)}{t N_{y} N_{z} a^{2}},
$$

where $N^{+}$and $N^{-}$are the total number of jumps along and against the electric field for a cross section slice in the $y z$ plane. We keep track of the time evolution of the current density $J(t)$ and stop the calculation when a converged current $J$ has been obtained. This usually takes several million hopping events. Note that following the standard practice we exclude from the calculations $N_{\text {in }}$ initial hopping events (typically $N_{i n} \sim 10^{4}$ ) [26] because they can give an extreme contribution to the current and thus lead to incorrect $J$.

(vi) Calculation of the conductivity and the Seebeck coefficient. The conductivity $\sigma$ is obtained from $\sigma=\frac{J}{F}$, where the electric field $F=\frac{V}{L_{x}}$. The Seebeck coefficient is computed according to Eq. (5), which requires a computation

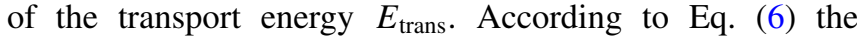
transport energy defines the energy of states that conduct the current most efficiently [22]. In our calculations we store the energy levels over which carriers jump and determine $E_{\text {trans }}$ from the analysis of the current density $J$ as a function of the energy. Note that in the calculation of the current and the transport energy we exclude a contribution of so-called soft jumps where charges are trapped for a long time in the pairs of spatially and energetically close sites [52].

Finally, we have confirmed a validity of the implemented Monte Carlo algorithm by comparing the calculated results with the available data in the literature [25].
[1] O. Bubnova and X. Crispin, Energy Environ. Sci. 5, 9345 (2012).

[2] Y. Xuan, X. Liu, S. Desbief, P. Leclere, M. Fahlman, R. Lazzaroni, M. Berggren, J. Cornil, D. Emin, and X. Crispin, Phys. Rev. B 82, 115454 (2010).

[3] O. Bubnova, M. Berggren, and X. Crispin, J. Am. Chem. Soc. 134, 16456 (2012).

[4] O. Bubnova, Z. U. Khan, A. Malti, S. Braun, M. Fahlman, M. Berggren, and X. Crispin, Nat. Mater. 10, 429 (2011).

[5] T. Park, C. Park, B. Kim, H. Shin, and E. Kim, Energy Environ. Sci. 6, 788 (2013)

[6] T.-C. Tsai, H.-C. Chang, C.-H. Chen, Y.-C. Huang, and W.-T. Whang, Org. Electronics 15, 641 (2014).

[7] M. Culebras, C. M. Gmez, and A. Cantarero, Materials 7, 6701 (2014).

[8] L. Groenendaal, F. Jonas, D. Freitag, H. Pielartzik, and J. R. Reynolds, Adv. Mater. 12, 481 (2000).

[9] M. V. Fabretto, D. R. Evans, M. Mueller, K. Zuber, P. Hojati-Talemi, R. D. Short, G. G. Wallace, and P. J. Murphy, Chem. Mater. 24, 3998 (2012).

[10] O. Bubnova, Z. U. Khan, H. Wang, S. Braun, D. R. Evans, M. Fabretto, P. Hojati-Talemi, D. Dagnelund, J.-B. Arlin, Y. H. Geerts, S. Desbief, D. W. Breiby, J. W. Andreasen, R. Lazzaroni, W. Chen, I. Zozoulenko, M. Fahlman, P. J. Murphy, M. Berggren, and X. Crispin, Nat. Mater. 13, 190 (2014).

[11] S. Stafsröm, Chem. Soc. Rev. 39, 2484 (2010).

[12] J. Hwang, D. B. Tanner, I. Schwendeman, and J. R. Reynolds, Phys. Rev. B 67, 115205 (2003).

[13] S. K. M. Jönsson, J. Birgerson, X. Crispin, G. Greczynskib, W. Osikowicz, A. W. Denier van der Gon, W. R. Salaneck, M. Fahlman, Synth. Met. 139, 1 (2003).

[14] X. Crispin, F. L. E. Jakobsson, A. Crispin, P. C. M. Grim, P. Andersson, A. Volodin, C. van Haesendonck, M. Van der Auweraer, W. R. Salaneck, and M. Berggren, Chem. Mater. 18, 4354 (2006).

[15] A. M. Nardes, M. Kemerink, R. A. J. Janssen, J. A. M. Bastiaansen, N. M. M. Kiggen, B. M. W. Langeveld, A. J. J. M. van Breemen, and M. M. de Kok, Adv. Mater. 19, 1196 (2007),

[16] A. M. Nardes, R. A. J. Janssen, and M. Kemerink, Adv. Funct. Mater. 18, 865 (2008).
[17] A. M. Nardes, M. Kemerink, and R. A. J. Janssen, Phys. Rev. B 76, 085208 (2007).

[18] A. J. Kronemeijer, E. H. Huisman, I. Katsouras, P. A. van Hal, T. C. T. Geuns, P. W. M. Blom, S. J. van der Molen, and D. M. de Leeuw, Phys. Rev. Lett. 105, 156604 (2010).

[19] N. Kim, B. H. Lee, D. Choi, G. Kim, H. Kim, J.-R. Kim, J. Lee, Y. H. Kahng, and K. Lee, Phys. Rev. Lett. 109, 106405 (2012).

[20] D. Nilsson, M. Chen, T. Kugler, T. Remonen, M. Armgarth, and M. Berggren, Adv. Mater. 14, 54 (2002).

[21] H. Sirringhaus, Adv. Mater. 17, 2411 (2005).

[22] R. Schmechel, J. Appl. Phys. 93, 4653 (2003).

[23] G. Kim and K. P. Pipe, Phys. Rev. B 86, 085208 (2012).

[24] S. D. Baranovskii, Phys. Status Solidi B 251, 487 (2014).

[25] H. Bässler, Phys. Status Solidi B 175, 15 (1993).

[26] J. J. M. van der Holst, F. W. A. van Oost, R. Coehoorn, and P. A. Bobbert, Phys. Rev. B 83, 085206 (2011)

[27] J. Cottaar, R. Coehoorn, and P. A. Bobbert, Organic Electronics 13, 667 (2012)

[28] D. Mendels and N. Tessler, J. Appl. Phys. 117, 105502 (2015).

[29] N. W. Ashcroft and N. D. Mermin, Solid State Physics (Sounders College, Fort Worth, 1976).

[30] D. Emin, in Wiley Encyclopedia of Electrical and Electronics Engineering Online, edited by J. G. Webster (John Wiley \& Sons, New York, 2002), pp. 1-44.

[31] B. I. Shklovskii, A. L. Efros, Electronic properties of doped semiconductors (Springer, Heidelberg, 1984).

[32] O. Madelung, Inroduction to Solid-State Theory (Springer, Heidelberg, 1996).

[33] A. Miller and E. Abrahams, Phys. Rev. 120, 745 (1960).

[34] M. C. J. M. Vissenberg and M. Matters, Phys. Rev. B 57, 12964 (1998); J. Nelson, ibid. 67, 155209 (2003); N. Sedghi, D. Donaghy, M. Raja, S. Badriya, S. Higgins, and W. Eccleston, J. Non-Cryst. Solids 352, 1641 (2006); M. Estrada, I. Mejia, A. Cerdeira, J. Pallares, L. Marsal, and B. Iniguez, Solid State Electron. 52, 787 (2008); M. Tachiya and K. Seki, Phys. Rev. B 82, 085201 (2010)

[35] S. Roche and D. Mayou, Phys. Rev. Lett. 79, 2518 (1997).

[36] F. Triozon, S. Roche, A. Rubio, and D. Mayou, Phys. Rev. B 69, 121410(R) (2004). 
[37] L. Li, N. Lu, and M. Liu, Europhys. Lett. 106, 17005 (2014).

[38] R. Coehoorn, W. F. Pasveer, P. A. Bobbert, and M. A. J. Michels, Phys. Rev. B 72, 155206 (2005).

[39] K. P. Pernstich, B. Rössner, and B. Batlogg, Nat. Mater. 7, 321 (2008).

[40] K. Harada, M. Sumino, C. Adachi, S. Tanaka, and K. Miyazaki, Appl. Phys. Lett. 96, 253304 (2010).

[41] F. C. Lavarda, M. C. dos Santos, D. S. Galvao, and B. Laks, Phys. Rev. B 49, 979 (1994).

[42] D. Giri and K. Kundu, Phys. Rev. B 53, 4340 (1996).

[43] A. Dkhissi, D. Beljonne, and R. Lazzaroni, F. Louwet, L. Groenendaal, and J. L. Brédas, Int. J. Quantum Chem. 91, 517 (2003).

[44] A. Dkhissi, D. Beljonne, and R. Lazzaroni, Synth. Met. 159, 546 (2009).
[45] M. Leufgen, O. Rost, C. Gould, G. Schmidt, J. Geurts, L. W. Molenkamp, N. S. Oxtoby, M. Mas-Torrent, N. Crivillers, J. Veciana, and C. Rovira, Organic Electronics 9, 1101 (2008).

[46] J. O. Oelerich, D. Huemmer, and S. D. Baranovskii, Phys. Rev. Lett. 108, 226403 (2012).

[47] B. D. Paulsen and C. D. Frisbie, J. Phys. Chem. C 116, 3132 (2012).

[48] S. Wang, M. Ha, M. Manno, C. D. Frisbie, and C. Leighton, Nat. Commun. 3, 1210 (2012).

[49] E.-G. Kim and J.-L. Brédas, J. Am. Chem. Soc. 130, 16880 (2008).

[50] A. Lenz, H. Kariis, A. Pohl, P. Persson, and L. Ojamäe, Chem. Phys. 384, 44 (2011).

[51] M. Jakobsson and S. Stafström, J. Chem. Phys. 135, 134902 (2011).

[52] A. M. Somoza and M. Ortuño, Phys. Rev. B 72, 224202 (2005). 